\title{
AN EXPLICIT FORMULA FOR THE SPLITTING OF MULTIPLE EIGENVALUES FOR NONLINEAR EIGENVALUE PROBLEMS, AND CONNECTIONS WITH THE LINEARIZATION FOR THE DELAY EIGENVALUE PROBLEM
}

\author{
WIM MICHIELS*, ISLAM BOUSSAADA, AND SILVIU-IULIAN NICULESCU
}

\begin{abstract}
We contribute to the perturbation theory of nonlinear eigenvalue problems in three ways. First, we extend the formula for the sensitivity of a simple eigenvalue with respect to a variation of a parameter to the case of multiple non-semisimple eigenvalues, thereby providing an explicit expression for the leading coefficients of the Puiseux series of the emanating branches of eigenvalues. Secondly, for a broad class of delay eigenvalue problems, the connection between the finite-dimensional nonlinear eigenvalue problem and an associated infinite-dimensional linear eigenvalue problem is emphasized in the developed perturbation theory. Finally, in contrast to existing work on analyzing multiple eigenvalues of delay systems, we develop all theory in a matrix framework, i.e., without reduction of problem to the analysis of a scalar characteristic quasi-polynomial.
\end{abstract}

Keywords. nonlinear eigenvalue problems, systems of functional equations and inequalities, perturbations of nonlinear operators, asymptotic distribution of eigenvalues and eigenfunctions, matrix and operator equations.

AMS Subject Classification: 35P30, 39B72, 47H14, 35P20, 39B42.

1. Introduction. Eigenvalue analysis and computations are important in many fields of science and engineering, and they also play an important role in the performance of many numerical algorithms. The context of the present paper is a generalization of the standard eigenvalue problem, known as the nonlinear eigenvalue problem, characterized by a nonlinear dependence of the characteristic matrix on the eigenvalue parameter. This type of eigenvalue problem includes quadratic eigenvalue problems [26], polynomial and rational eigenvalue problems, as well as eigenvalue problems inferred from the analysis of delay differential equations [22]. A short overview of properties of generic nonlinear eigenvalue problems can be found in [28] and the references therein.

In this paper we consider the nonlinear eigenvalue problem

$$
M(\lambda ; \epsilon) v=0, \lambda \in \mathbb{C}, v \in \mathbb{C}^{n}, v \neq 0,
$$

parameterized by a real parameter $\epsilon$, and focus on the behavior of an eigenvalue $\lambda_{0}$ for $\epsilon=0$ (i.e., $\operatorname{det} M\left(\lambda_{0} ; 0\right)=0$ ), as a function of $\epsilon$ We assume there exist an open set $\Omega \subset \mathbb{C}$, containing $\lambda_{0}$, and an open interval $I \subset \mathbb{R}$, containing zero, such that the following properties are satisfied: for all $\epsilon \in I$ the entries of $M$ are analytic functions of $\lambda$ on $\Omega$, and for all $\lambda \in \Omega$ the entries of $M$ are smooth functions of $\epsilon$ on $I$. Furthermore, for all $\epsilon \in I$, we assume that $M$ is not singular (in the sense of singularity of a pencil [15]), i.e., $\operatorname{det}(M(\lambda ; \epsilon)) \not \equiv 0$. We aim to contribute to the perturbation theory of nonlinear eigenvalue problems in three ways.

First, we extend the formula for the sensitivity of a simple eigenvalue with respect to a variation of the parameter to the case of multiple non-semisimple eigenvalues, satisfying a complete regular splitting property. Here, we contribute to the key references $[13,16,17,18,19]$ by providing an explicit expression for the leading coefficients of the Puiseux series of the eigenvalue, in terms of eigenvectors and generalized eigenvectors. Second, for a broad class of delay eigenvalue problems, the connection between the

\footnotetext{
* Department of Computer Science, Katholieke Universiteit Leuven, Celestijnenlaan 200A, 3001 Heverlee, Belgium (Wim.Michiels@cs.kuleuven.be).
} 
finite-dimensional nonlinear eigenvalue problem and an associated infinite-dimensional linear eigenvalue problem, which has been instrumental in the design of algorithms for eigenvalue computations [30, 14], is further developed for the case of multiple eigenvalues, with inclusion of the perturbation theory developed in the first part of the paper. Finally, we point out that the existing studies of multiple eigenvalues of delay equations almost exclusively rely on the analysis of roots of a scalar characteristic quasi-polynomial. We quote the functional structured matrix approach characterizing multiple roots of [2], the conceptually appealing approach for eigenvalue perturbation analysis for time-delay systems with commensurate delays established in [4], and the geometric/sectorial approach established in [8] for time-delay systems where the delays are not necessarily commensurate, but under a non-degeneracy assumption. The approach we develop in the present work relies on a matrix framework, avoiding the reduction to a scalar characteristic quasi-polynomials.

Even though multiple eigenvalues are non-generic, in the sense that they occur with probability zero if elements of the matrices are chosen randomly, there are special situations that favor their presence. The first situation corresponds to optimized spectra using stability optimization techniques, which are important in the context of control design. The minimization of the spectral abscissa or spectral radius typically gives rises to multiple non-semisimple active eigenvalues $[27,3]$, resulting in a non-locally Lipschitz objective function. Secondly, double non-semisimple eigenvalues on the imaginary axis play a major role in distance problems for eigenvalue problems with Hamiltonian symmetry, which occur in the context of optimal control and $\mathcal{H}_{\infty}$ computations [9]. Finally, multiple semisimple eigenvalues appear in problems characterized by symmetries in dynamical systems or networks. For instance, in [6] a network composed from four identical Brusselator chemical reactors is considered, where the existence of multi-dimensional irreducible representations of the symmetry group may force an eigenvalue to be multiple. Such an intriguing phenomenon were subject of a huge bibliography in quest of characterizing special properties for behavior of eigenvalues with symmetry like gyroscopic or conservative systems, see for instance [24]

We conclude by briefly rehearsing some basic concepts, introducing notation and presenting some motivating examples, one of which will be used throughout the paper. The algebraic multiplicity of an eigenvalue (1.1) is equal to the multiplicity of this eigenvalue as a root of the characteristic equation

$$
\operatorname{det} M(\lambda ; \epsilon)=0
$$

while the geometric multiplicity equals the dimension of the null space of $M\left(\lambda_{0} ; \epsilon\right)$. In this paper we study perturbations of an eigenvalue $\lambda_{0}$ of (1.1) for $\epsilon=0$ with algebraic multiplicity $m$ and geometric multiplicity one. Vectors $H_{0}, \ldots H_{m-1}$ that satisfy

$$
H_{0} \neq 0, \quad \sum_{k=0}^{l} \frac{1}{k !} \frac{\partial^{k} M}{\partial \lambda^{k}}\left(\lambda_{0} ; 0\right) H_{l-k}=0, \quad l=0, \ldots, m-1 .
$$

form the corresponding Jordan chain. In what follows we use subscripts for partial derivatives $\left(M_{\lambda}:=\frac{\partial M}{\partial \lambda}, M_{\epsilon}=: \frac{\partial M}{\partial \epsilon}\right)$, and to simplify the notation, we omit the 
argument $\left(\lambda_{0} ; 0\right)$ when it is clear from the context. In this way (1.3) is spelled out as

$$
\begin{aligned}
M H_{0} & =0, \quad H_{0} \neq 0, \\
M H_{1}+\frac{1}{1 !} M_{\lambda} H_{0} & =0, \\
M H_{2}+\frac{1}{1 !} M_{\lambda} H_{1}+\frac{1}{2 !} M_{\lambda \lambda} H_{0} & =0, \\
\vdots & \vdots \\
M H_{m-1}+\frac{1}{1 !} M_{\lambda} H_{m-2}+\cdots+\frac{1}{(m-1) !} M_{\lambda^{m-1}} H_{0} & =0 .
\end{aligned}
$$

For more information about spectral properties of analytic matrix functions, excellent references are [28, 13]. See also [29] and the references therein for the computation of Jordan chains. To illustrate the importance of taking the nonlinearity of the eigenvalue problem into account, consider

$$
M_{1}(\lambda)=\left[\begin{array}{cc}
\lambda & 1 \\
e^{-\lambda}-1 & \lambda
\end{array}\right]
$$

having zero as a simple eigenvalue, even though $M(0)$ has the form of a two-by-two Jordan block. In the same spirit,

$$
M_{2}(\lambda ; \epsilon)=\left[\begin{array}{cc}
\lambda-1+e^{-\lambda}+\epsilon & 0 \\
0 & \lambda+1+\epsilon
\end{array}\right]
$$

has for $\epsilon=0$ zero as a double, non-semisimple eigenvalue, with Jordan chain

$$
H_{0}=\left[\begin{array}{l}
1 \\
0
\end{array}\right], H_{1}=\left[\begin{array}{l}
\alpha \\
0
\end{array}\right]
$$

for any $\alpha \in \mathbb{R}$, even though $M(0)$ is a diagonal matrix. This example also illustrate that generalized eigenvectors do not need to be linear independent [28]. In the nonlinear case, even the null vector is allowed as generalized eigenvector.

The structure of the paper is as follows. In Section 2 we derive as main result an explicit expression for the leading coefficients in the Puiseux expansion of a multiple non-semisimple eigenvalue satisfying the completely regular splitting property, in terms of left and right eigenvectors and generalized eigenvectors. This formula generalizes the well know expression for the sensitivity of a simple eigenvalue. In Section 3 we apply the results to a broad class of time-delay systems. In addition we rephrase Jordan chains and the sensitivity formula in terms of an equivalent linear operator eigenvalue problem, which can be seen as a linearization of the nonlinear delay eigenvalue problem. In Section 4 we provide some illustrations of the presented results, and in Section 5 we present the main conclusions.

2. Sensitivity formula for multiple eigenvalues. We start with a technical lemma about the parameterization of a Jordan chain.

LEMMA 2.1. Let $\lambda_{0}$ be an eigenvalue of (1.1) for $\epsilon=0$, with algebraic multiplicity equal to $m$ and geometric multiplicity one, and let $\left(\hat{H}_{0}, \ldots, \hat{H}_{m-1}\right)$ be a particular Jordan chain. Then all possible Jordan chains can be parameterized as

$$
\begin{aligned}
\left(H_{0}, \ldots, H_{m-1}\right)= & \alpha_{0}\left(\hat{H}_{0}, \ldots, \hat{H}_{m-1}\right)+\alpha_{1}\left(0, \hat{H}_{0}, \ldots, \hat{H}_{m-2}\right) \\
& +\alpha_{2}\left(0,0, \hat{H}_{0}, \ldots, \hat{H}_{m-3}\right)+\ldots+\alpha_{m-1}\left(0, \ldots, 0, \hat{H}_{0}\right),
\end{aligned}
$$

with $\alpha_{k} \in \mathbb{C}, k=0, \ldots, m$ and $\alpha_{0} \neq 0$. 
Proof. It is clear that the eigenvector can be parameterized by $H_{0}=\alpha_{0} \hat{H}_{0}$. We now solve

$$
M H_{1}=-M_{\lambda}\left(\alpha_{0} \hat{H}_{0}\right) .
$$

for $H_{1}$. Clearly $\alpha_{0} \hat{H}_{1}$ is a solution since $\left(\hat{H}_{0}, \hat{H}_{1}\right)$ is part of a Jordan chain. Moreover, since the null space of $M$ is one-dimensional, with null vector $\hat{H}_{0}$, the set of all solutions is parameterized by

$$
H_{1}=\alpha_{0} \hat{H}_{1}+\alpha_{1} \hat{H}_{0}
$$

with $\alpha_{1}$ an arbitrary complex scalar. Let us now solve the equation

$$
M H_{2}=-M_{\lambda}\left(\alpha_{0} \hat{H}_{1}+\alpha_{1} \hat{H}_{0}\right)-\frac{1}{2} M_{\lambda \lambda}\left(\alpha_{0} \hat{H}_{0}\right) .
$$

A particular solution is given by $\alpha_{0} \hat{H}_{2}+\alpha_{1} \hat{H}_{1}$. This follows from substituting the expression, grouping terms by $\alpha_{0}$ and $\alpha_{1}$, and using the fact that $\left(\hat{H}_{0}, \hat{H}_{1}, \hat{H}_{2}\right)$ is part of a Jordan chain. Once again, since $\hat{H}_{0}$ is the sole null vector of $M$, all solutions are given by

$$
H_{2}=\alpha_{0} \hat{H}_{2}+\alpha_{1} \hat{H}_{1}+\alpha_{2} \hat{H}_{0}
$$

with $\alpha_{2}$ an arbitrary complex scalar. Repeating this argument leads to the assertion to be proven.

The main result of this section is contained in the following theorem.

THEOREM 2.2. Let $\lambda_{0}$ be an eigenvalue of (1.1) for $\epsilon=0$, with algebraic multiplicity equal to $m$ and geometric multiplicity one, with Jordan chain $\left(H_{0}, \ldots, H_{m-1}\right)$. Let $U_{0}$ be the corresponding left eigenvector. Assume that condition

$$
U_{0}^{*} M_{\epsilon} H_{0} \neq 0
$$

holds. Then around $\epsilon=0$, the eigenvalues in the vicinity of $\lambda_{0}$ can be expanded as the branches of the Puiseux series

$$
\lambda(\epsilon)=\lambda_{0}+\sum_{i=1}^{\infty} \epsilon^{\frac{i}{m}} \lambda_{i}
$$

where

$$
\lambda_{1}^{m}=-\frac{U_{0}^{*} M_{\epsilon} H_{0}}{U_{0}^{*}\left(\frac{1}{1 !} M_{\lambda} H_{m-1}+\frac{1}{2 !} M_{\lambda \lambda} H_{m-2}+\cdots+\frac{1}{m !} M_{\lambda^{m}} H_{0}\right)} .
$$

Proof. Because of the assumed multiplicities and (2.2), we have a completely regular splitting property [13]. It follows that the eigenvalue functions can be expanded as (2.3), with $\lambda_{1} \neq 0$. Furthermore, the corresponding eigenvectors can be expanded as

$$
v(\epsilon)=V_{0}+\sum_{i=1}^{\infty} \epsilon^{\frac{i}{m}} V_{i},
$$

see [16, Lemma 2]. By definition we have

$$
M(\lambda(\epsilon) ; \epsilon) v(\epsilon)=0 .
$$


Substituting (2.3), (2.5), and working out this expression in increasing powers of $\epsilon / \mathrm{m}$ gives:

$$
\begin{array}{ll}
\text { (0) } & M V_{0}=0 \\
\text { (1) } & M V_{1}+\frac{1}{1 !} \frac{\partial M}{\partial \lambda} \lambda_{1} V_{0}=0 \\
\text { (2) } & M V_{2}+\frac{1}{1 !} \frac{\partial M}{\partial \lambda}\left(\lambda_{1} V_{1}+\lambda_{2} V_{0}\right)+\frac{1}{2 !} \frac{\partial^{2} M}{\partial \lambda^{2}} \lambda_{1}^{2} V_{0}=0 \\
\text { (3) } & M V_{3}+\frac{1}{1 !} \frac{\partial M}{\partial \lambda}\left(\lambda_{1} V_{2}+\lambda_{2} V_{1}+\lambda_{3} V_{0}\right)+\frac{1}{2 !} \frac{\partial^{2} M}{\partial \lambda^{2}}\left(\lambda_{1}^{2} V_{1}+2 \lambda_{1} \lambda_{2} V_{0}\right) \\
& +\frac{1}{3 !} \frac{\partial^{3} M}{\partial \lambda^{3}} \lambda_{1}^{3} V_{0}=0 \\
\text { (4) } & M V_{4}++\frac{1}{1 !} \frac{\partial M}{\partial \lambda}\left(\lambda_{1} V_{3}+\lambda_{2} V_{2}+\lambda_{3} V_{1}+\lambda_{4} V_{0}\right) \\
& +\frac{1}{2 !} \frac{\partial^{2} M}{\partial \lambda^{2}}\left(\lambda_{1}^{2} V_{2}+2 \lambda_{1} \lambda_{2} V_{1}+\left(\lambda_{2}^{2}+2 \lambda_{1} \lambda_{3}\right) V_{0}\right) \\
& +\frac{1}{3 !} \frac{\partial^{3} M}{\partial \lambda^{3}}\left(\lambda_{1}^{3} V_{1}+3 \lambda_{1}^{2} \lambda_{2} V_{0}\right)++\frac{1}{4 !} \frac{\partial^{4} M}{\partial \lambda^{4}} \lambda_{1}^{4} V_{0}=0 \\
& \vdots \\
\text { (i) } & M V_{i}+\frac{1}{1 !} \frac{\partial M}{\partial \lambda} C_{i, 1}+\cdots+\frac{1}{i !} \frac{\partial^{2} M}{\partial \lambda^{2}} C_{i, i}=0 \\
& \vdots \\
(m-1) & M V_{m-1}+\frac{1}{1 !} \frac{\partial M}{\partial \lambda} C_{m-1,1}+\cdots+\frac{1}{m_{m-1)}} \frac{\partial^{m-1} M}{\partial \lambda^{m-1}} C_{m-1, m-1}=0 \\
\text { (m) } & M V_{m}+\frac{1}{1 !} \frac{\partial M}{\partial \lambda} C_{m, 1}+\cdots+\frac{1}{m !} \frac{\partial^{m} M}{\partial \lambda^{m}} C_{m, m}+\frac{\partial M}{\partial \epsilon} V_{0}=0,
\end{array}
$$

where the vectors $C_{i, j}$ are linear combinations of $\left(V_{0}, \ldots, V_{i-j}\right)$, explicitly expressed as



Multiplying the last equation of (2.6) from the left with $U_{0}^{*}$ and taking into account that

$$
U_{0}^{*} M=0, \quad V_{0}=\frac{C_{m, m}}{\lambda_{1}^{m}}
$$

we arrive at

$$
\lambda_{1}^{m}=-\frac{U_{0}^{*} \frac{\partial M}{\partial \epsilon} C_{m, m}}{U_{0}^{*}\left(\frac{\partial M}{\partial \lambda} C_{m, 1}+\cdots+\frac{1}{m !} \frac{\partial^{m} M}{\partial \lambda^{m}} C_{m, m}\right)} .
$$

Subsequently, we show that $\left(C_{m, m}, \ldots, C_{m, 1}\right)$ forms a Jordan chain. The condition

$$
M C_{m, m}=0
$$

is obtained by multiplying expression (0) in (2.6) with $\lambda_{1}^{m}$. Similarly, condition

$$
M C_{m, m-1}+\frac{1}{1 !} \frac{\partial M}{\partial \lambda} C_{m, m}=0
$$

is obtained by taking a linear combination of expression (0) and (1) with coefficients $(m-1) \lambda_{2} \lambda_{1}^{m-2}$ and $\lambda_{1}^{m-1}$. More generally, for $\ell \in\{0, \ldots, m-1\}$ condition

$$
M C_{m, m-\ell}+\frac{1}{1 !} \frac{\partial M}{\partial \lambda} C_{m, m-\ell+1}+\ldots+\frac{1}{\ell !} \frac{\partial^{\ell} M}{\partial \lambda^{\ell}} C_{m, m}
$$


is obtained by a linear combination of expression $(0),(1), \ldots,(\ell)$, with coefficients given by



So far we have established that the coefficient vectors $\left(C_{m, m}, \ldots, C_{m, 1}\right)$, appearing in (2.8), form a particular Jordan chain. Because a Jordan chain is not unique we can complete the proof by showing that formula (2.8) is independent of the choice of the Jordan chain. Following Lemma 2.1, all Jordan chains can be parameterized as

$$
\begin{aligned}
\left(J_{m}, \ldots, J_{1}\right)= & \alpha_{0}\left(C_{m, m}, \ldots, C_{m, 1}\right)+\alpha_{1}\left(0, C_{m, m}, \ldots, C_{m, 2}\right)+\alpha_{2}\left(0,0, C_{m, m}, \ldots, C_{m, 3}\right) \\
& +\cdots+\alpha_{m-1}\left(0, \ldots 0, C_{m, m}\right),
\end{aligned}
$$

where $\alpha_{i} \in \mathbb{C}, i=0, \ldots, m-1$ and $\alpha_{0} \neq 0$. By the definition of a Jordan chain, we get from this equation

$$
\begin{aligned}
U_{0}^{*}\left(\frac{\partial M}{\partial \lambda} J_{1}+\cdots+\frac{1}{m !} \frac{\partial^{m} M}{\partial \lambda^{m}} J_{m}\right)= & \alpha_{0} U_{0}^{*}\left(\frac{\partial M}{\partial \lambda} C_{m, 1}+\cdots+\frac{1}{m !} \frac{\partial^{m} M}{\partial \lambda^{m}} C_{m, m}\right) \\
& +U_{0}^{*}\left(-\alpha_{1} M C_{m, 1}-\alpha_{2} M C_{m, 2}-\cdots\right. \\
& \left.-\alpha_{m-1} M C_{m, m-1}\right) \\
= & \alpha_{0} U_{0}^{*}\left(\frac{\partial M}{\partial \lambda} C_{m, 1}+\cdots+\frac{1}{m !} \frac{\partial^{m} M}{\partial \lambda^{m}} C_{m, m}\right)
\end{aligned}
$$

Since, in addition, we have $J_{m}=\alpha_{0} C_{m, m}$, we arrive at

$$
\frac{U_{0}^{*} \frac{\partial M}{\partial \epsilon} C_{m, m}}{U_{0}^{*}\left(\frac{\partial M}{\partial \lambda} C_{m, 1}+\cdots+\frac{1}{m !} \frac{\partial^{m} M}{\partial \lambda^{m}} C_{m, m}\right)}=\frac{U_{0}^{*} \frac{\partial M}{\partial \epsilon} J_{m}}{U_{0}^{*}\left(\frac{\partial M}{\partial \lambda} J_{1}+\cdots+\frac{1}{m !} \frac{\partial^{m} M}{\partial \lambda^{m}} J_{m}\right)} .
$$

Choosing $\left(J_{1}, \ldots, J_{m}\right)=\left(H_{m-1}, \ldots, H_{0}\right)$, and substituting $(2.9)$ in $(2.8)$ we arrive at (2.4).

REMARK 2.3. When switching the role of $\lambda$ and $\epsilon$, condition (2.2) can be interpreted as if the eigenvalue were simple.

Remark 2.4. For the special case where $m=1$, Lemma 2.7 of [23] is recovered.

Example 1. Consider example (1.4). In addition to (1.5), we have at $\lambda=0$ and $\epsilon=0$,

$$
U_{0}=\left[\begin{array}{l}
1 \\
0
\end{array}\right], M_{\epsilon}=I, M_{\lambda}=\left[\begin{array}{ll}
0 & 0 \\
0 & 1
\end{array}\right], M_{\lambda \lambda}=\left[\begin{array}{ll}
1 & 0 \\
0 & 0
\end{array}\right]
$$

Substituting these values in (2.8), we obtain

$$
\lambda_{1}=\sqrt{-2} .
$$

An interesting observation about the coefficients $C_{i j}$ in (2.6) is that they satisfy a recursion relation, as expressed in the following proposition. 
Proposition 2.5. The vectors $C_{i, j}$ in the proof of Theorem 2.2 satisfy the following $2 D$-recurrence:

$$
\left\{\begin{aligned}
C_{i, 1} & =\sum_{l=1}^{i} \lambda_{l} V_{i-l} \quad \text { for } \quad 1 \leq i \leq m \\
C_{1, j} & =0 \quad \text { for } \quad 2 \leq j \leq m \\
C_{i, j} & =\sum_{l=1}^{i-1} \lambda_{l} C_{i-l, j-1} \quad \text { for } \quad 2 \leq i, j \leq m .
\end{aligned}\right.
$$

Proof. The formulae given in (2.11) represent symbolically vectors $C_{i, j}$ as the components of a block triangular matrix $C$. The proof of the third equality from (2.11) is by induction, initialized by the first two equalities from (2.11).

Example 2. For $m=4$, matrices $C_{i, j}$ take the following form:

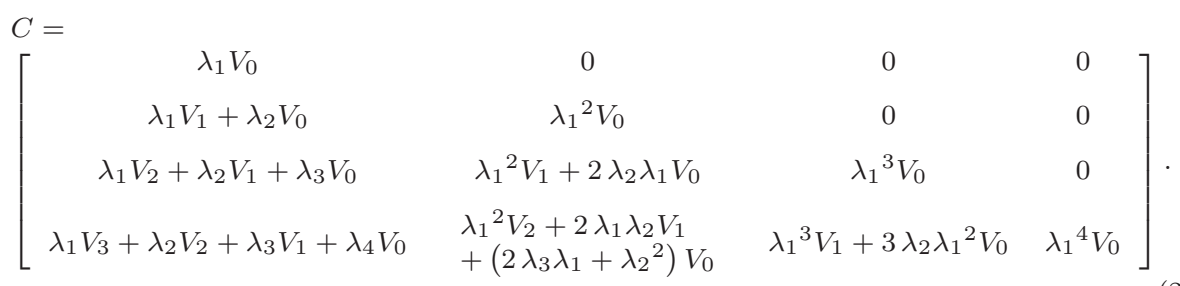

We end the section with an alternative proof of Theorem 2.2. This proof is based on the explicit construction of the local Smith form, which is the starting point of the analysis in $[17,18,19]$.

Alternative proof of Theorem 2.2. From the Jordan chain we can construct a root function of order $m$, following the lines of [7],

$$
\phi(\lambda)=H_{0}+H_{1}\left(\lambda-\lambda_{0}\right)+\cdots+H_{m-1}\left(\lambda-\lambda_{0}\right)^{m-1} H_{m-1} .
$$

Let $X \in \mathbb{C}^{n \times(n-1)}$ be such that matrix $\left[H_{0} X\right]$ has full rank. This property and the property that the geometric multiplicity of eigenvalue $\lambda_{0}$ is one, imply that $M\left(\lambda_{0} ; 0\right) X$ has full column rank. Using the definition of a Jordan chain, we get

$$
\begin{aligned}
M(\lambda ; 0)[\phi(\lambda) X] & =\left(M+\sum_{k=1}^{\infty} \frac{1}{k !} M_{\lambda^{k}}\left(\lambda-\lambda_{0}\right)^{k}\right)[\phi(\lambda) X] \\
& =\left[\Xi\left(\lambda-\lambda_{0}\right)^{m}+\left(\lambda-\lambda_{0}\right)^{m+1} G(\lambda) M(\lambda ; 0) X\right],
\end{aligned}
$$

with vector $\Xi$ given by

$$
\Xi=\frac{1}{m !} M_{\lambda^{m}} H_{0}+\frac{1}{(m-1) !} M_{\lambda^{m-1}} H_{1}+\cdots+\frac{1}{1 !} M_{\lambda} H_{m-1}
$$

and function $G$ analytic. It follows that

$$
M(\lambda ; 0)[\phi(\lambda) X]=\left[\Xi+\left(\lambda-\lambda_{0}\right) G(\lambda) M(\lambda ; 0) X\right]\left[\begin{array}{ll}
\left(\lambda-\lambda_{0}\right)^{m} & \\
& I
\end{array}\right] .
$$

In the neighborhood of $\lambda_{0}$, matrix $[\phi(\lambda) X]$ is invertible, hence, we can express

$$
M(\lambda ; 0)=\underbrace{\left[\Xi+\left(\lambda-\lambda_{0}\right) G(\lambda) M(\lambda ; 0) X\right]}_{E(\lambda)} \underbrace{\left[\begin{array}{c}
\left(\lambda-\lambda_{0}\right)^{m} \\
I
\end{array}\right]}_{D(\lambda)} \underbrace{[\phi(\lambda) X]^{-1}}_{F(\lambda)} .
$$


In addition, also the above matrix $E(\lambda)$ is invertible near $\lambda_{0}$, which can be shown by contradiction: the condition $\Xi=M\left(\lambda_{0} ; 0\right) X \beta$ for some $\beta \in \mathbb{C}^{n-1}$, along with (2.13), would namely imply that the Jordan chain would have length larger than $m$. We conclude that the factorization (2.14) corresponds to a local Smith form of $M(\lambda ; 0)$ at $\lambda=\lambda_{0}$.

We are now ready to use the results of [17] in which the leading coefficients of the Puiseux series are obtained from the Newton diagram of function $\operatorname{det} M$, and expressed in terms of the local Smith form. More precisely from Corollary 1 of [17] it can be derived, for the problem under consideration, that

$$
\lambda_{1}^{m}=-e_{1}^{T} E\left(\lambda_{0}\right)^{-1} \frac{\partial M}{\partial \epsilon}\left(\lambda_{0} ; 0\right) F\left(\lambda_{0}\right)^{-1} e_{1},
$$

with $e_{1}$ the first unit vector in $\mathbb{R}^{n}$.

Using the expression for the Smith form we get

$$
F\left(\lambda_{0}\right)^{-1} e_{1}=\phi\left(\lambda_{0}\right)=H_{0} .
$$

Second, we have to determine $v, v^{*}=e_{1}^{T} E\left(\lambda_{0}\right)^{-1}$. This correspond to solving the system of equations

$$
v^{*} E\left(\lambda_{0}\right)=e_{1}^{T} \Leftrightarrow v^{*} \Xi=1, v^{*} M\left(\lambda_{0} ; 0\right) X=0 .
$$

Recall from the above that $\left[\Xi M\left(\lambda_{0} ; 0\right) X\right]$ is invertible, and there is a unique solution. As a consequence we must have $U_{0}^{*} \Xi \neq 0$, and a solution is given by

$$
v=\frac{1}{\Xi^{*} U_{0}} U_{0}
$$

Substituting (2.16) and (2.17) in (2.15) yields (2.4).

3. Reformulation in terms of linearization for delay eigenvalue problem. We apply the results of Section 2 to general linear functional differential equations of the form

$$
\dot{x}(t)=\int_{-\tau_{\max }}^{0} d \mu(\theta) x(t+\theta), \quad x(t) \in \mathbb{C}^{n},
$$

where $\mu$ has bounded variation in $\left[-\tau_{\max }, 0\right]$ and satisfies $\mu(0)=0$. We note that a very broad class of time-delay systems can be brought into this form [11]. For instance, if we let

$$
0=\tau_{0}<\tau_{1}<\cdots<\tau_{k} \leq \tau_{\max }
$$

and define

$$
\begin{cases}\mu(0) & =0 \\ \mu(\theta) & =-\sum_{i=0,-\tau_{i}>\theta}^{k} A_{i}, \quad \theta \in\left(-\tau_{\max }, 0\right) \\ \mu\left(-\tau_{\max }\right) & =-\sum_{i=0}^{k} A_{i},\end{cases}
$$

where $A_{i}$ is a n-by-n matrix for, $0 \leq i \leq k$, then (3.1) becomes

$$
\dot{x}(t)=A_{0} x(t)+\sum_{i=1}^{k} A_{i} x\left(t-\tau_{i}\right),
$$


an equation featuring pointwise (discrete) delay. Similarly, if we let

$$
\left\{\begin{array}{ll}
\mu(0) & =0 \\
\mu(\theta) & =-\int_{\theta}^{0} A(s) d s, \\
\mu\left(-\tau_{\max }\right) & =-\int_{-\tau_{\max }}^{0} A(s) d s,
\end{array} \quad \theta \in\left(-\tau_{\max }, 0\right)\right.
$$

with $A$ a continuous function from $\left[-\tau_{\max }, 0\right]$ to $\mathbb{C}^{n \times n}$, we end up with an equation with distributed (continuous) delay,

$$
\dot{x}(t)=\int_{-\tau_{\max }}^{0} A(\theta) x(t+\theta) d \theta .
$$

As we shall see in Section 3.1, the nonlinear eigenvalue problem related to (3.1), i.e., (1.1) with

$$
M(\lambda):=\lambda I-\int_{-\tau_{\max }}^{0} d \mu(\theta) e^{\lambda \theta},
$$

can be rephrased as an linear, infinite-dimensional operator eigenvalue problem. Our goal is to express all results of the previous section in terms of this operator eigenvalue problem. This will lead us to equivalent expressions for Jordan chains (Section 3.2) and for the sensitivity of eigenvalues (Section 3.3), which are completely similar to the expressions for the matrix eigenvalue problem. The generalization lies in the fact that matrices are replaced by operators, and vector-vector products by an appropriate bilinear form over two function spaces. Finally, in Section 3.4 we briefly comment on the allowable algebraic multiplicity for the case of discrete delays.

3.1. Reformulation as an infinite-dimensional linear system. The initial condition for the time-delay system (3.1) is a function segment

$$
\phi \in \mathcal{C}\left(\left[-\tau_{\max }, 0\right], \mathbb{C}^{n}\right),
$$

where $\mathcal{C}\left(\left[-\tau_{\max }, 0\right], \mathbb{C}^{n}\right)$ is the space of continuous functions mapping the interval $\left[-\tau_{\max }, 0\right]$ into $\mathbb{C}^{n}$. For every $\phi \in \mathcal{C}\left(\left[-\tau_{\max }, 0\right], \mathbb{C}^{n}\right)$ the forward system of $(3.1)$ is uniquely defined $[11,12]$. We denote by

$$
x(\phi): t \in\left[-\tau_{\max }, \infty\right) \rightarrow x(\phi)(t) \in \mathbb{C}^{n}
$$

the solution with initial conditions $\phi$, i.e.,

$$
x(\phi)(\theta)=\phi(\theta), \forall \theta \in\left[-\tau_{\max }, 0\right]
$$

The state at time $t$, in the sense of information needed to uniquely continue the solution beyond time $t$, is given by the function segment $x_{t}(\phi) \in \mathcal{C}\left(\left[-\tau_{\max }, 0\right], \mathbb{C}^{n}\right)$,

$$
x_{t}(\phi)(\theta)=x(\phi)(t+\theta), \theta \in\left[-\tau_{\max }, 0\right] .
$$

In order to reformulate (3.1) as a linear system in standard form, we follow the approach of [5]. When we define the infinite-dimensional space

$$
X:=\mathbb{C}^{n} \times \mathcal{L}_{2}\left(\left[-\tau_{\max }, 0\right], \mathbb{C}^{n}\right)
$$


and the linear operator $\mathcal{A}$ as

$$
\begin{aligned}
& \mathcal{D}(\mathcal{A})=\left\{(x ; \phi) \in X: \phi^{\prime} \in \mathcal{L}_{2}\left(\left[-\tau_{\max }, 0\right], \mathbb{C}^{n}\right), \quad x=\phi(0)\right\}, \\
& \mathcal{A}(x ; \phi)=\left(\int_{-\tau_{\max }}^{0} d \mu(\theta) \phi(\theta) ; \phi^{\prime}\right),
\end{aligned}
$$

we can rewrite (3.1) as an ordinary differential equation on $X$,

$$
\frac{d}{d t} z(t)=\mathcal{A} z(t)
$$

Note that $\mathcal{A}$ is the infinitesimal operator of the semi-group of solution operators associated with (3.1). The connections between solutions of (3.1) and (3.3) are as follows. The decomposition $z=\left(z_{1} ; z_{2}\right)$ can be interpreted as an arrow, where the "head" $z_{1}(t)$ corresponds to the current value $x(t)$, while the "tail" $z_{2}(t)$ corresponds to the history $x_{t}(t)$ over an interval of length $\tau_{\max }$.

The substitution of a sample solution of the form $e^{\lambda t} v$ in $(3.1)$, with $v \in \mathbb{C}^{n} \backslash\{0\}$ leads us to the nonlinear eigenvalue problem

$$
M(\lambda) v=0
$$

where $M$ is given by (3.2). The equation

$$
\operatorname{det} M(\lambda)=0
$$

is called the characteristic equation of (3.1) and its roots the characteristic roots. These are related to the spectrum of the linear operator $\mathcal{A}$ in the following way. The characteristic roots are the eigenvalues of the operator $\mathcal{A}$, which only features a point spectrum, that is,

$$
\sigma(\mathcal{A})=\operatorname{P\sigma }(\mathcal{A})
$$

Furthermore, the algebraic multiplicity of a complex number $\lambda$ as an eigenvalue of $\mathcal{A}$ is equal to its multiplicity as a root of the characteristic equation, while its geometric multiplicity is equal to the dimension of the null space of $M(\lambda)$, see [22]. These properties are important because they are compatible with the definition of multiplicities of solutions of the nonlinear eigenvalue problem (3.4). For more details about the connection between the eigenvalue problems we refer to [22].

In summary, equation (3.1) can brought into a first order form (3.3). In accordance, the nonlinear eigenvalue problem (3.4) and (3.2) can be reformulated as the operator eigenvalue problem

$$
(\lambda I-\mathcal{A}) \mathcal{V}=0, \mathcal{V} \in X, \mathcal{V} \neq 0
$$

Note that (3.5) can be seen as a linearization of (3.4) and (3.2), in the same way as a quadratic eigenvalue problem (second order system) can be reformulated as a linear eigenvalue problem (first order system) of doubled dimension. The difference is that the step to be taken is not from dimension $n$ to $2 n$ but from dimension $n$ to $\infty$.

3.2. Corresponding Jordan chain for operator $\mathcal{A}$. In the following result, we connect a Jordan chain of the nonlinear eigenvalue problem (3.4) with the Jordan chain of the linear (infinite-dimensional) eigenvalue problem for operator $\mathcal{A}$. 
THEOREM 3.1. If $\lambda_{0}$ is an eigenvalue of (3.4) with Jordan chain $\left(H_{0}, \ldots, H_{m-1}\right)$, then the functions $\left(\mathcal{H}_{0}, \ldots, \mathcal{H}_{m-1}\right)$ defined as

$$
\begin{aligned}
\mathcal{H}_{0} & =\left(H_{0} ; H_{0} e^{\lambda_{0} \theta}, \theta \in\left[-\tau_{\max }, 0\right]\right), \\
\mathcal{H}_{1} & =\left(H_{1} ;\left(H_{1}+\theta H_{0}\right) e^{\lambda_{0} \theta}, \theta \in\left[-\tau_{\max }, 0\right]\right), \\
& \vdots \\
\mathcal{H}_{m-1} & =\left(H_{m-1} ;\left(H_{m-1}+\theta H_{m-2}+\frac{\theta^{2}}{2 !} H_{m-3}+\cdots+\frac{\theta^{m-1}}{(m-1) !} H_{0}\right) e^{\lambda_{0} \theta},\right.
\end{aligned}
$$

form a Jordan chain of the operator $\mathcal{A}$ corresponding to the eigenvalue $\lambda_{0}$, i.e. they satisfy

$$
\begin{array}{ll}
\left(\mathcal{A}-\lambda_{0} I\right) & \mathcal{H}_{0}=0 \\
\left(\mathcal{A}-\lambda_{0} I\right) & \mathcal{H}_{k}=\mathcal{H}_{k-1}, \quad 1 \ldots, m-1 .
\end{array}
$$

Furthermore, (3.1) has solutions of the form

$$
x(t)=\sum_{i=0}^{m-1} c_{i}\left(H_{i}+\frac{t}{1 !} H_{i-1}+\cdots+\frac{t^{i}}{i !} H_{0}\right) e^{\lambda_{0} t},
$$

where $c_{0}, \ldots, c_{m-1}$ are arbitrary complex numbers.

Conversely, let the functions $\left(\mathcal{H}_{0}, \ldots, \mathcal{H}_{m-1}\right)$ form a Jordan chain of operator $\mathcal{A}$, corresponding eigenvalue $\lambda_{0}$. Then these functions take form (3.6), where $\left(H_{0}, \ldots, H_{m-1}\right)$ is a Jordan chain of the nonlinear eigenvalue problem $M(\lambda) v=0$, corresponding $\lambda_{0}$.

Proof. We start with the first assertion. Decompose (3.6) as $\mathcal{H}_{k}=\left(H_{k} ; \mathcal{H}_{k, 2}\right), k=$ $0, \ldots, m-1$. By construction $\mathcal{H}_{k} \in \mathcal{D}(\mathcal{A})$. From

$$
\begin{aligned}
& \lambda_{0} H_{k}+H_{k-1}-\int_{-\tau_{\max }}^{0} d \mu(\theta) \mathcal{H}_{k, 2}(\theta) \\
& =\lambda_{0} H_{k}+H_{k-1}-\int_{-\tau_{\max }}^{0} d \mu(\theta)\left(H_{k}+\theta H_{k-1}+\cdots+\frac{\theta^{k}}{(k) !} H_{0}\right) e^{\lambda_{0} \theta} \\
& =M\left(\lambda_{0}\right) H_{k}+\frac{M^{\prime}\left(\lambda_{0}\right)}{1 !} H_{k-1}+\cdots+\frac{1}{k !} \frac{d^{k} M\left(\lambda_{0}\right)}{d \lambda^{k}} H_{0}=0,
\end{aligned}
$$

it follows that (3.7) holds what concerns the first component. For the second component we have, by construction,

$$
\mathcal{H}_{k, 2}^{\prime}-\lambda_{0} \mathcal{H}_{k, 2}=\left\{\begin{array}{ll}
\mathcal{H}_{k-1}, & k=1, \ldots, m-1, \\
0 & k=0 .
\end{array},\right.
$$

and the proof of (3.7) is complete.

Substituting (3.8) into (3.1) yields

$$
e^{\lambda_{0} t} \sum_{i=0}^{m-1} c_{i} \sum_{k=0}^{i} \frac{t^{i-k}}{(i-k) !}\left(M\left(\lambda_{0}\right) H_{k}+\frac{M^{\prime}\left(\lambda_{0}\right)}{1 !} H_{k-1}+\cdots+\frac{1}{k !} \frac{d^{k} M\left(\lambda_{0}\right)}{d \lambda^{k}} H_{0}\right)=0 .
$$

This equation is satisfied for all $t \geq 0$ because $\left(H_{0}, \ldots, H_{m-1}\right)$ is a Jordan chain for (3.4). The second assertion follows.

Finally, we prove the converse result step-by-step. Consider equation

$$
\left(\mathcal{A}-\lambda_{0} I\right) \mathcal{H}_{0}=0 .
$$


With $\mathcal{H}_{0}:=\left(\mathcal{H}_{0,1} ; \mathcal{H}_{0,2}\right)$ we get

$$
\mathcal{H}_{0,2}^{\prime}=\lambda_{0} \mathcal{H}_{0,2} \Rightarrow \mathcal{H}_{0,2}=e^{\lambda_{0} \theta} H_{0},
$$

for some vector $H_{0}$, as well as

$$
\lambda_{0} H_{0}=\lambda_{0} \mathcal{H}_{0,1}=\int_{-\tau_{\max }}^{0} d \mu(\theta) \mathcal{H}_{0,2}(\theta)=\int_{-\tau_{\max }}^{0} d \mu(\theta) e^{\lambda_{0} \theta} H_{0},
$$

which corresponds to

$$
M\left(\lambda_{0}\right) H_{0}=0 .
$$

Next we analyze equation

$$
\left(\mathcal{A}-\lambda_{0} I\right) \mathcal{H}_{1}=\mathcal{H}_{0} .
$$

Considering the second component leads us to the differential equation

$$
\mathcal{H}_{1,2}^{\prime}(\theta)=\lambda_{0} \mathcal{H}_{1,2}(\theta)+e^{\lambda_{0} \theta} H_{0},
$$

with explicit solution of the form

$$
\mathcal{H}_{1,2}(\theta)=\left(H_{1}+\theta H_{0}\right) e^{\lambda_{0} \theta} .
$$

Considering the first component leads us to

$$
H_{0}+\lambda_{0} H_{1}=\int_{-\tau_{\max }}^{0} d \mu(\theta) H_{1} e^{\lambda_{0} \theta}+\int_{-\tau_{\max }}^{0} d \mu(\theta) \theta H_{0} e^{\lambda_{0} \theta},
$$

which can be rephrased as

$$
M\left(\lambda_{0}\right) H_{1}+\frac{1}{1 !} M^{\prime}\left(\lambda_{0}\right) H_{0}=0 .
$$

Expressions (3.9) and (3.10) correspond to a Jordan chain of $M$ of length at least two. Repeating this argument leads to the assertion of the proposition.

EXAMPLE 3. Let us come back to the nonlinear eigenvalue problem described by (1.4). For $\epsilon=0$, this eigenvalue problem corresponds to delay system

$$
\dot{x}(t)=\left[\begin{array}{rr}
1 & 0 \\
0 & -1
\end{array}\right] x(t)+\left[\begin{array}{cc}
-1 & 0 \\
0 & 0
\end{array}\right] x(t-1),
$$

which is of the form (3.1), with $\tau_{\max }=1$ and

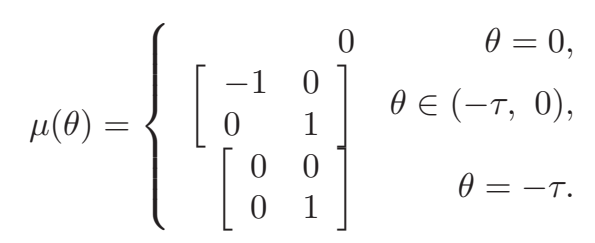

For this problem operator $\mathcal{A}$ reads as

$$
\begin{aligned}
& \mathcal{D}(\mathcal{A})=\left\{(x ; \phi) \in X: \phi^{\prime} \in \mathcal{L}_{2}\left(\left[-\tau_{\max }, 0\right], \mathbb{C}^{2}\right), \quad x=\phi(0)\right\} \\
& \mathcal{A}(x ; \phi)=\left(\left[\begin{array}{cc}
1 & 0 \\
0 & -1
\end{array}\right] \phi(0)+\left[\begin{array}{cc}
-1 & 0 \\
0 & 0
\end{array}\right] \phi(-1) ; \quad \phi^{\prime}\right)
\end{aligned}
$$


and the Jordan chain for eigenvalue zero, corresponding to (1.5) is given by

$$
\begin{aligned}
& \mathcal{H}_{0}=\left(\left[\begin{array}{l}
1 \\
0
\end{array}\right] ;\left[\begin{array}{l}
1 \\
0
\end{array}\right]\right), \\
& \left.\mathcal{H}_{1}=\left(\begin{array}{l}
\alpha \\
0
\end{array}\right] ;\left[\begin{array}{l}
\alpha+\theta \\
0
\end{array}\right], \theta \in[-1,0]\right) .
\end{aligned}
$$

We check, for $\lambda_{0}=0$,

$$
\begin{aligned}
& \left(\mathcal{A}-\lambda_{0} I\right) \mathcal{H}_{1}=\left(\left[\begin{array}{l}
\alpha-(\alpha-1) \\
0 \\
1-1 \\
0
\end{array}\right] ;\left[\begin{array}{l}
1 \\
0 \\
0
\end{array}\right]\right)=0 .
\end{aligned}
$$

3.3. Corresponding formula for the sensitivity. In this section we rephrase the result of Theorem 2.2 in terms of linear operator $\mathcal{A}$ and its eigenfunctions. Consider the space

$$
X^{*}:=\mathbb{C}^{1 \times n} \times \mathcal{L}_{2}\left([0, \tau], \mathbb{C}^{1 \times n}\right)
$$

and the following bilinear form on $X^{*} \times X$,

$$
\langle\Psi, \Phi\rangle:=\mu \nu+\int_{-\tau_{\max }}^{0} \int_{\theta}^{0} \psi(\xi-\theta) d \eta(\theta) \phi(\xi) d \xi,
$$

with $\Psi=(\mu ; \psi) \in X^{*}$ and $\Phi=(\nu ; \phi) \in X$. In [11, Section 7] the formal adjoint of $\mathcal{A}$ with respect to this bilinear form was derived, and properties of this operator were investigated. It follows from the analysis in [11] that the left eigenfunction $\mathcal{U}_{0} \in X^{*}$ of $\mathcal{A}$ corresponding to $\lambda_{0}$ is given by

$$
\mathcal{U}_{0}:=\left(U_{0}^{*} ; U_{0}^{*} e^{-\lambda_{0} \theta}, \theta \in\left[0, \tau_{\max }\right]\right),
$$

and that left and right eigenfunctions corresponding to different eigenvalues are orthogonal with respect to the bilinear form (3.13).

Let us now make the dependence on parameter $\epsilon$ explicit, that is, we study

$$
\dot{x}(t)=\int_{-\tau_{m}}^{0} d \mu(\theta, \epsilon) x(t+\theta),
$$

and its characteristic equation

$$
M(\lambda ; \epsilon)=\lambda I-\int_{-\tau_{\max }}^{0} d \mu(\theta, \epsilon) e^{\lambda \theta},
$$

where we assume that the function

$$
I \ni \epsilon \mapsto \int_{-\tau_{\max }}^{0} d \mu(\theta, \epsilon) \phi(\theta)
$$

is defined for every $\phi \in \mathcal{C}\left(\left[-\tau_{\max }, 0\right], \mathbb{C}^{n}\right)$ and smooth whenever $\phi$ is smooth. The "derivative" of operator $\mathcal{A}$ with respect to $\epsilon$ can then be expressed by

$$
\begin{aligned}
& \mathcal{D}\left(\mathcal{A}_{\epsilon}\right)=\left\{(x ; \phi) \in X: \phi^{\prime} \in \mathcal{L}_{2}\left(\left[-\tau_{\max }, 0\right], \mathbb{C}^{n}\right), \quad x=\phi(0)\right\}, \\
& \mathcal{A}_{\epsilon}(x ; \phi)=\left(\frac{d}{d \epsilon}\left(\int_{-\tau_{\max }}^{0} d \mu(\theta, \epsilon) \phi(\theta)\right) ; 0\right) .
\end{aligned}
$$


The result corresponding to Theorem 2.2 can now be expressed in the following way.

THEOREM 3.2. Let $M$ be given by (3.15). Assume that the conditions of Theorem 2.2 hold, and let $\mathcal{U}_{0}$ be defined by (3.14). Using the bilinear form (3.13), we can express

$$
\lambda_{1}^{m}=\frac{\left\langle\mathcal{U}_{0}, \mathcal{A}_{\epsilon} \mathcal{H}_{0}\right\rangle}{\left\langle\mathcal{U}_{0}, \mathcal{H}_{m-1}\right\rangle}
$$

Proof. Let us start with the nominator. Defining

$$
N(\lambda, \epsilon)=\int_{-\tau_{\max }}^{0} d \mu(\theta, \epsilon) e^{\lambda \theta}
$$

and suppressing the dependence on $\epsilon$ in the notation, we get

$$
\begin{aligned}
\left\langle U_{0}, \mathcal{H}_{m-1}\right\rangle= & U_{0}^{*} H_{m-1}+\int_{-\tau_{\max }}^{0} \int_{\theta}^{0} U_{0}^{*} e^{\lambda_{0} \theta} d \eta(\theta)\left(H_{m-1}+\frac{\xi}{1 !} H_{m-2}\right. \\
& \left.+\frac{\xi^{2}}{2 !} H_{m-3} \cdots+\frac{\xi^{m-1}}{(m-1) !} H_{0}\right) d \xi \\
= & U_{0}^{*} H_{m-1}-U_{0}^{*} \int_{-\tau_{\max }}^{0} e^{\lambda \theta} d \eta(\theta)\left(\frac{\theta}{1 !} H_{m-1}+\frac{\theta^{2}}{2 !} H_{m-2}\right. \\
& \left.\quad+\frac{\theta^{3}}{3 !} H_{m-3}+\cdots+\frac{\theta^{m}}{m !} H_{0}\right) \\
= & U_{0}^{*}\left(\left(I-N_{\lambda}\right) H_{m-1}-\frac{1}{2 !} N_{\lambda \lambda} H_{m-2}-\frac{1}{3 !} N_{\lambda^{3}} H_{m-3}-\ldots\right. \\
= & \left.-\frac{1}{m !} N_{\lambda^{m}} H_{0}\right) \\
= & U_{0}^{*}\left(\frac{1}{1 !} M_{\lambda} H_{m-1}+\frac{1}{2 !} M_{\lambda \lambda} H_{m-2}+\frac{1}{3 !} M_{\lambda^{3}} H_{m-3}+\ldots \frac{1}{m !} M_{\lambda^{m}} H_{0}\right) .
\end{aligned}
$$

Regarding the denominator, notice first that

$$
\frac{d}{d \epsilon} \int_{-\tau_{\max }}^{0} d \mu(\theta, \epsilon) e^{\lambda_{0} \theta} H_{0}=N_{\epsilon}\left(\lambda_{0} ; \epsilon\right) H_{0}=-M_{\epsilon}\left(\lambda_{0} ; \epsilon\right) H_{0}
$$

It readily follows, at $\epsilon=0$,

$$
\mathcal{A}_{\epsilon} \mathcal{H}_{0}=\left(-M_{\epsilon} H_{0} ; 0\right)
$$

and

$$
\left\langle\mathcal{U}_{0}, \mathcal{A}_{\epsilon} \mathcal{H}_{0}\right\rangle=-U_{0}^{*} M_{\epsilon} H_{0} .
$$

The assertion follows from a direct comparison with the expression in Theorem 2.2.

EXAMPLE 4. Let us, for the last time, come back to (1.4) and analyze the behavior of the zero eigenvalue as a function of $\epsilon$, around zero. The right eigenfunctions have been determined as (3.12). The left eigenfunction is

$$
\mathcal{U}_{0}=\left(\left[\begin{array}{ll}
1 & 0
\end{array}\right] ;\left[\begin{array}{ll}
1 & 0
\end{array}\right]\right)
$$

and $\mu(\theta ; \epsilon)$ satisfies

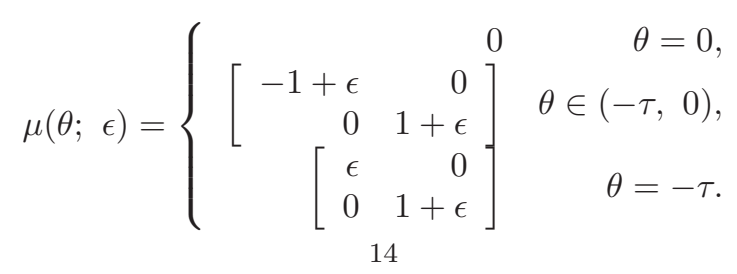


We have

$$
\mathcal{A}_{\epsilon}(x ; \phi)=(-\phi(0) ; 0)
$$

By direct substitution we get $\left\langle\mathcal{U}_{0}, \mathcal{A}_{\epsilon} \mathcal{H}_{0}\right\rangle=-1$. Moreover, we get

$$
\begin{aligned}
\left\langle\mathcal{U}_{0}, \mathcal{H}_{1}\right\rangle & =\alpha+\int_{-1}^{0} \int_{\theta}^{0}[10] d \mu(\theta)\left[\begin{array}{r}
\alpha+\xi \\
0
\end{array}\right] d \xi \\
& =\alpha+\int_{-1}^{0}\left[\begin{array}{ll}
1 & 0
\end{array}\right] d \mu(\theta)\left[\begin{array}{r}
-\alpha \theta-\frac{\theta^{2}}{2} \\
0
\end{array}\right] \\
& =\alpha+\left[\begin{array}{ll}
1 & 0
\end{array}\right]\left(\left[\begin{array}{rr}
1 & 0 \\
0 & -1
\end{array}\right]\left[\begin{array}{l}
0 \\
0
\end{array}\right]+\left[\begin{array}{rr}
-1 & 0 \\
0 & 0
\end{array}\right]\left[\begin{array}{r}
\alpha-\frac{1}{2} \\
0
\end{array}\right]\right)=\frac{1}{2} .
\end{aligned}
$$

Hence, an application of formula (3.16) gives $\lambda_{1}^{2}=-2$, which is consistent with (2.10).

REMARK 3.3. In the above framework delay sensitivity can be addressed as well. For this, note $\tau_{\max }$ can be chosen strictly larger than the actual largest delay. Even though this renders the state space non-minimal, it has no influence on the location of the eigenvalues of corresponding operator $\mathcal{A}$.

3.4. Note on the admissible multiplicities for systems with discrete delay. Since we are dealing with infinite-dimensional systems, we provide a characterization of admissible multiplicities for eigenvalues of time-delay systems. Consider the particular class of (3.1) with discrete delays only:

$$
\dot{x}(t)=A_{0} x(t)+\sum_{i=1}^{N} A_{i} x\left(t-\tau_{i}\right), \quad x(t) \in \mathbb{R}^{n},
$$

for which the corresponding characteristic function is given by

$$
\Delta(\lambda):=\operatorname{det}\left(\lambda I-A_{0}-\sum_{i=1}^{N} A_{i} e^{-\lambda \tau_{i}}\right) .
$$

Making the dependence on the delays explicit, this quasi-polynomial function can be written in the form

$$
\Delta(z, \tau)=P_{0}(z)+\sum_{k=1}^{\tilde{N}} P_{k}(z) e^{\sigma_{k} z}
$$

where $\sigma_{k}$ are admissible combinations of the components of the delay vector $\tau:=$ $\left(\tau_{1}, \ldots, \tau_{N}\right) ; \sigma_{k}:=-\sum_{l=1}^{N} \alpha_{k, l} \tau_{l}$ such that $0 \leq \alpha_{k, l} \leq n$, and $\tilde{N}$ is the cardinality of all admissible $\sigma_{k}$, which is a positive integer satisfying $\tilde{N} \geq N$.

Let the degree of the quasi-polynomial function (3.19) be defined as the number of the corresponding polynomials plus the sum of their respective degrees minus one. The following theorem provides a bound on the maximum multiplicity of an eigenvalue. Its proof is analogous to the ones of Proposition 5.1 in [1] and Lemma 1 in [2], where the special case of eigenvalues on the imaginary axis is addressed.

THEOREM 3.4. Consider system (3.17).

- The multiplicity of a real characteristic root is bounded by the degree of the corresponding quasi-polynomial function, the so-called Polya-Szegö bound. 
- The multiplicity of a non-real characteristic root is bounded by the number of non-vanishing coefficients of the delayed part of the corresponding quasipolynomial function.

Proof. Without any loss of generality, all the $\sigma_{k}$ in (3.19) are assumed to be distinct, and $\sigma:=\left(\sigma_{1}, \ldots, \sigma_{N}\right)$ is considered as an auxiliary delay vector for the quasi polynomial, see for instance [1,2]. It is also assumed that $a_{i, j}$ stands for the coefficient of the monomial $z^{j}$ in $P_{i}, a_{0}:=\left(a_{0,0}, \ldots, a_{0, n}\right)^{\top}$ is the vector composed from the coefficients of the polynomial $P_{0}$ and $p:=\left(a_{1,0}, \ldots, a_{1, n_{1}-1}, \ldots, a_{\tilde{N}, 0}, \ldots, a_{\tilde{N}, n_{\tilde{N}}-1}\right)^{\top} \in \mathbb{R}^{\eta}$. It follows from (3.18) that $P_{0}$ is a monic polynomial of degree $n$ in $z$ and the polynomials $P_{k}$ are such that $\operatorname{deg}\left(P_{k}\right):=d_{k}-1=n-\sum_{l=1}^{N} \alpha_{k, l} \leq(n-1)$.

A given complex number $z_{0}$ is an eigenvalue of (3.17) for some delay vector $\tau^{*}$ if $\Delta\left(z_{0}, \tau^{*}\right)=0$ We denote by $\partial_{z}^{k} \Delta(z, \tau)$ the $k$-th derivative of $\Delta(z, \tau)$ given by $(3.19)$ with respect to the variable $z$. A spectral value $z_{0}$ is of algebraic multiplicity $m \geq 1$ if $\Delta\left(z_{0}, \tau^{*}\right)=\partial_{z} \Delta\left(z_{0}, \tau^{*}\right)=\ldots=\partial_{z}^{m-1} \Delta\left(z_{0}, \tau^{*}\right)=0$ and $\partial_{z}^{m} \Delta\left(z_{0}, \tau^{*}\right) \neq 0$. The main ingredient of the remaining proof is to write the set of $\Delta\left(z_{0}, \tau^{*}\right)=\partial_{z} \Delta\left(z_{0}, \tau^{*}\right)=\ldots=$ $\partial_{z}^{m-1} \Delta\left(z_{0}, \tau^{*}\right)=0$ into a matrix representation considering $p$ as the variable vector and $\sigma$ as a vector parameter. Namely, with an appropriate construction the matrix corresponds to a functional structured matrix (see Appendix A). As a matter of fact, in the case of a multiple real root one obtains a functional Vandermonde matrix (A.1) with the corresponding function given by $g(x):=e^{\lambda_{0} x}$. The first $n-1$ equations of the obtained linear system (in $p$ ) gives $a_{0}$ as a function in $p$ and $\sigma$. The subsystem of equations from equation $n+1$ to equation $n+\eta$ gives the unique solution $p \equiv 0$, which is not consistent with the $n$-th equation. Thus, the maximal multiplicity of a given real root is nothing but $n+\eta$ which is the degree of the quasi-polynomial.

The proof of the second assertion is also based on the construction of a linear system from $\Delta\left(z_{0}, \tau^{*}\right)=\partial_{z} \Delta\left(z_{0}, \tau^{*}\right)=\ldots=\partial_{z}^{m-1} \Delta\left(z_{0}, \tau^{*}\right)=0$ involving trigonometric Vandermonde matrices as the ones established in [2]. The remaining proof is in the same lines as the ones of Lemma 1 in [2].

\section{Numerical examples.}

4.1. Multiple eigenvalues on the imaginary axis. Consider the planar timedelay system with an uncertain delay $\tilde{\tau}_{2}=\tau_{2}+\epsilon$ :

$$
\left\{\begin{array}{l}
\dot{x}_{1}=x_{2}, \\
\dot{x}_{2}=-x_{1}(t)-a x_{1}\left(t-\tau_{1}\right)-b x_{1}\left(t-\left(\tau_{2}+\epsilon\right)\right),
\end{array}\right.
$$

The characteristic matrix corresponding to (4.1) is given by

$$
M(\lambda ; \epsilon)=\left[\begin{array}{cc}
\lambda & -1 \\
1+a \mathrm{e}^{-\tau_{1} \lambda}+b \mathrm{e}^{-\lambda\left(\tau_{2}+\epsilon\right)} & \lambda
\end{array}\right]
$$

for which the corresponding quasi-polynomial function reads as:

$$
\Delta(\lambda ; \epsilon)=\lambda^{2}+1+a \mathrm{e}^{-\lambda \tau_{1}}+b \mathrm{e}^{-\lambda\left(\tau_{2}+\epsilon\right)} .
$$


For $\epsilon=0$, a double imaginary axis eigenvalue $\lambda=j \omega$ occurs if the following equalities are simultaneously satisfied:

$$
\left\{\begin{array}{l}
\cos \left(\omega \tau_{1}\right)=-\frac{\tau_{2}\left(\omega^{2}-1\right)}{\left(\tau_{1}-\tau_{2}\right) a} \\
\cos \left(\omega \tau_{2}\right)=\frac{\tau_{1}(\omega-1)(\omega+1)}{b\left(\tau_{1}-\tau_{2}\right)} \\
\sin \left(\omega \tau_{1}\right)=-2 \frac{\omega}{\left(\tau_{1}-\tau_{2}\right) a} \\
\sin \left(\omega \tau_{2}\right)=2 \frac{\omega}{b\left(\tau_{1}-\tau_{2}\right)}
\end{array}\right.
$$

Consider $a, b, \tau_{1}, \tau_{2}$ and $\omega$ as variables, we have 4 equality constraints in 5 variables, and inequality constraints $\tau_{1} \geq 0, \tau_{2} \geq 0$. In what follows, we fix the parameter values according to the following solution.

\begin{tabular}{|c|c|c|c|c|c|}
\hline Parameter & $\omega$ & $\tau_{1}$ & $\tau_{2}$ & $a$ & $b$ \\
\hline Value & 4.5077329 & 0.096858286 & 1 & 23.606102 & 10.195100 \\
\hline
\end{tabular}

Next, we study the behavior of the double root as a function of $\epsilon$ around $\epsilon=0$. The eigenvalue is non-semisimple and the Jordan chain is given by:

$$
H_{0}=\left[\begin{array}{c}
1 \\
4.5077 j
\end{array}\right], H_{1}=\left[\begin{array}{c}
0.0966 j \\
0.5644
\end{array}\right] .
$$

The left eigenvector satisfies $U_{0}^{*}=\left[\begin{array}{ll}0.5 & 0.1109 j\end{array}\right]^{T}$. Using Theorem 2.2 and matrices

$$
\begin{aligned}
M_{\lambda}\left(\lambda_{0}, 0\right) & =\left[\begin{array}{cc}
1 & 0 \\
-9.0154 j & 1
\end{array}\right], \\
M_{\lambda \lambda}\left(\lambda_{0}, 0\right) & =\left[\begin{array}{cc}
0 & 0 \\
-1.8712+9.8886 j & 0
\end{array}\right], \\
M_{\epsilon}\left(\lambda_{0}, 0\right) & =\left[\begin{array}{cc}
0 & 0 \\
44.9977+9.3398 j & 0
\end{array}\right],
\end{aligned}
$$

we obtain $\lambda_{1}=1.9087+2.3770 j$. The solid lines in Figure 4.1 correspond to the first two terms in the expansion of the eigenvalues, following from Theorem 2.2, i.e.,

$$
\lambda^{(k)}(\epsilon)=j \omega \pm \lambda_{1} \epsilon^{\frac{1}{2}}, k \in\{1,2\} .
$$

for $\epsilon \in[-0.1,0.1]$ (positive blue, negative red). The circles are the eigenvalues computed with method [30] for $\epsilon$ values on an equidistant grid with gridsize 0.01 .

4.2. Spectral abscissa optimization. We analyze the model problem for spectral abscissa optimization used in, among others, [21, 27]. Consider the unstable system

$$
\dot{x}(t)=A x(t)+B u(t-\tau),
$$

with

$$
A=\left[\begin{array}{rrr}
-0.08 & -0.03 & 0.2 \\
0.2 & -0.04 & -0.005 \\
-0.06 & -0.2 & -0.07
\end{array}\right], B=\left[\begin{array}{r}
-0.1 \\
-0.2 \\
0.1
\end{array}\right], \tau=5
$$






FIG. 4.1. Migration of a double non-semisimple eigenvalue on the imaginary axis

which we wish to stabilize by static state feedback,

$$
u(t)=K^{T} x(t)=\left[\begin{array}{lll}
k_{1} & k_{2} & k_{3}
\end{array}\right] x(t) .
$$

The closed-loop system is of the form (3.1) with

$$
\tau_{\max } \leq \tau, \mu(\theta)=\left\{\begin{array}{cl}
0 & \theta=0, \\
-A & \theta \in(-\tau, 0), \\
-A-B K^{T} & \theta \leq-\tau,
\end{array}\right.
$$

and the corresponding eigenvalue problem is described by

$$
M(\lambda)=\lambda I-A-B K^{T} e^{-\lambda \tau} .
$$

Minimizing the spectral abscissa, i.e., the real part of the rightmost eigenvalue, leads us to the optimal gain values

$$
k_{1}=0.4 .7121273, k_{2}=0.50372106, k_{3}=0.6 .0231834 .
$$

Minimizing the spectral abscissa favor multiple roots. In this case, the optimum is characterized by a multiple root $\lambda_{\mathrm{opt}}=-0.14949804$ with algebraic multiplicity four and geometric multiplicity one. A Jordan chain for the closed loop system corresponding $\lambda_{\mathrm{opt}}$, and the left eigenvector are given by

$$
\left[\begin{array}{llll}
H_{0} & H_{1} & H_{2} & H_{3}
\end{array}\right]=\left[\begin{array}{rrrr}
0.9815 & -0.5932 & 1.9150 & 9.0174 \\
-0.1765 & -1.3604 & 13.2818 & 49.6470 \\
0.0740 & 4.6241 & 6.2759 & -1.2048
\end{array}\right], U_{0}=\left[\begin{array}{l}
0.6595 \\
0.5729 \\
0.4866
\end{array}\right] \text {. }
$$


Let us now investigate the sensitivity of the closed loop system with respect to the delay, $\tau=5+\epsilon$, for $\epsilon \in[-0.5,0.5]$. The formula for the sensitivity from Theorem 2.2 gives

$$
\lambda_{1}=(-0.00077380)^{\frac{1}{6}} .
$$

The solid lines in Figure 4.2, once again, correspond to the first two terms in the expansion of the eigenvalues, following from Theorem 2.2,

$$
\lambda^{(k)}(\epsilon)=\lambda_{\text {opt }}+\lambda_{1}^{\frac{1}{4}} \epsilon^{\frac{1}{m}} e^{j k \pi / 4}, k \in\{0,1,2,3\},
$$

for $\epsilon \in[-1 / 1200,1 / 1200]$ (positive blue, negative red). The circles are the eigenvalues for $\epsilon$ values on an equidistant grid with gridsize $1 / 1200$.

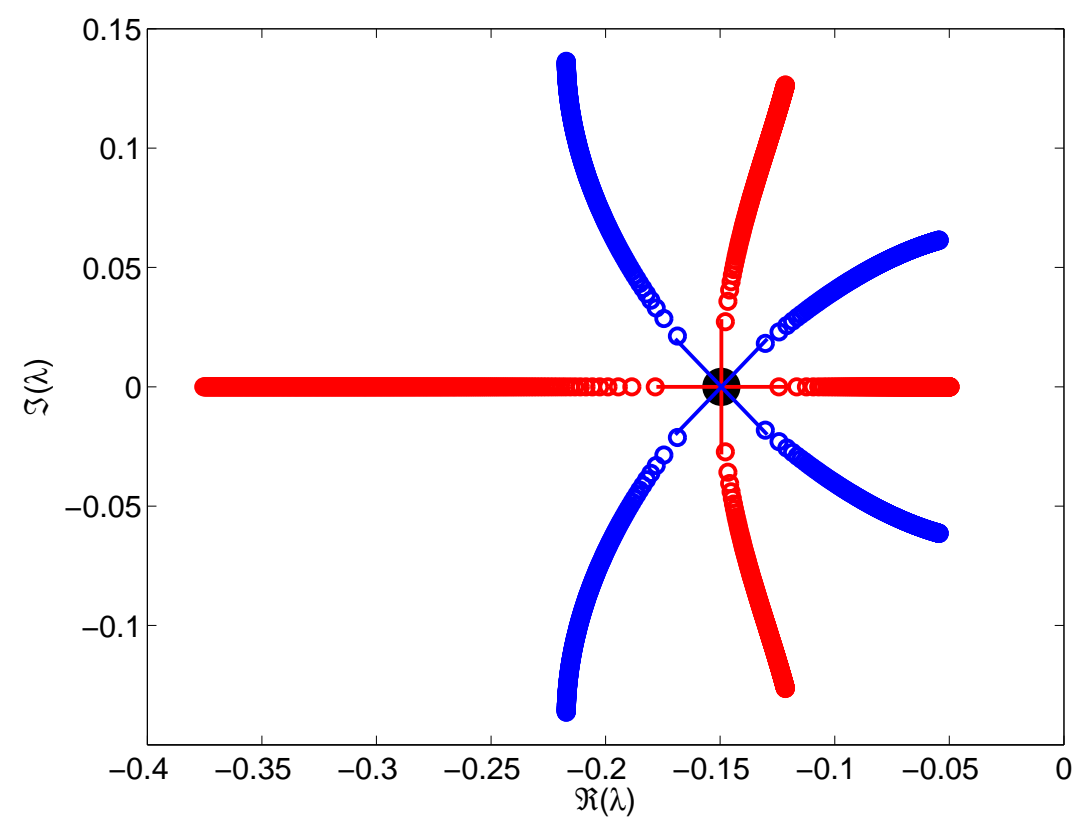

FIG. 4.2. Behavior of a real eigenvalue with multiplicity four, corresponding to a minimum of the spectral abscissa function, as a function of a delay parameter.

5. Conclusions. The main contributions of the paper lie in the sensitivity formula of Theorem 2.2, and in the "dual" treatment of the delay eigenvalue problem, where the Jordan chains and the sensitivity formula are addressed both at the level of the finite-dimensional nonlinear eigenvalue problem, and at the level of a standard operator eigenvalue problem.

Recall that the class of delay systems (3.1) is very broad, including both systems with discrete and distributed delay. The sensitivity formula in Theorem 3.2 is extremely simple, and it has the same form as for the standard (matrix) eigenvalue problem. It should be said, however, that, besides the eigenvectors, information about the system is present in the bilinear form (3.13) as well.

An interesting question is whether a result like Theorem 2.2 also applies to the most general case, where the geometric multiplicity of the eigenvalue can be larger 
than one, starting from a canonical system of Jordan chains. Probably the answer is yes, but the analysis is far from trivial. The first issue is the condition for a regular splitting property. The condition in Theorem 4.2 of [13] is expressed in terms of generating eigenvectors (limits of eigenvectors when the parameter approaches the critical value), which depend on the splitting behavior if the nullspace of $M$ has dimension larger than one. Secondly, even if a formula like (2.4) in Theorem 2.2 would remain valid, it is not clear which left eigenvector to select. The use of generating eigenvectors could lead to a circular argument: in order to characterize the splitting behavior we need the generating eigenvectors, but to obtain the generating eigenvectors, we need to know the splitting behavior.

Acknowledgements. The authors like to thank the anonymous reviewers for the constructive comments. They are particularly grateful for pointing to the work of Langer and Najman, which allowed them to provide a second proof of Theorem 2.2 based on the construction of the local Smith form.

The work of WM was supported by the Programme of Interuniversity Attraction Poles of the Belgian Federal Science Policy Office (IAP P6-DYSCO), by OPTEC, the Optimization in Engineering Center of the KU Leuven, by the project G.0712.11N of the Research Foundation-Flanders (FWO - Vlaanderen), and by the project UCoCoS, funded by the European Union's Horizon 2020 research and innovation programme under the Marie Sklodowska-Curie Grant Agreement No 675080. The work of IB and SIN is financially supported by CNRS, CentraleSupelec and IPSA. The authors are with the CNRS GDRI SPaDisCo European research network. Last but not least, the authors thank Luca Fenzi for the careful reading of the manuscript and the feedback.

\section{REFERENCES}

[1] I. Boussaada and S-I. Niculescu. Characterizing the codimension of zero singularities for timedelay systems: A link with Vandermonde and Birkhoff incidence matrices. To appear in: Acta Applicandae Mathematicae, pages 1-46, 2016.

[2] I. Boussaada and S-I. Niculescu. Tracking the algebraic multiplicity of crossing imaginary roots for generic quasipolynomials: A vandermonde-based approach. To appear in: IEEE Transactions on Automatic Control, page 6pp, 2016.

[3] J. V. Burke, A. S. Lewis, and M. L. Overton. Two numerical methods for optimizing matrix stability. Linear Algebra and its Applications, 351-352:117-145, 2002.

[4] J. Chen, P. Fu, S.-I. Niculescu, and Z. Guan. An eigenvalue perturbation approach to stability analysis, part i: Eigenvalue series of matrix operators. SIAM Journal on Control and Optimization, 48(8):5564-5582, 2010.

[5] R. F. Curtain and H. Zwart. An introduction to infinite-dimensional linear systems theory, volume 21 of Texts in Applied Mathematics. Springer Verlag, 1995.

[6] M. Dellnitz and B. Werner. Computational methods for bifurcation problems with symmetrieswith special attention to steady state and hopf bifurcation points. Journal of Computational and Applied Mathematics, 26(1-2):97 - 123, 1989.

[7] I. Gohberg, M.A. Kaashoek, and F. van Schagen. On the local theory of regular analytic matrix functions. Linear Algebra and its Applications, 182:9-25, 1993.

[8] K. Gu, D. Irofti, I. Boussaada, and S. I. Niculescu. Migration of double imaginary characteristic roots under small deviation of two delay parameters. In 2015 54th IEEE Conference on Decision and Control (CDC), pages 6410-6415, 2015.

[9] S. Gumussoy and W. Michiels. Fixed-order H-infinity control for interconnected using delay differential algebraic equations. SIAM Journal on Control and Optimization, 49(5):2212$2238,2011$.

[10] T.T. Ha and J.A. Gibson. A note on the determinant of a functional confluent Vandermonde matrix and controllability. Linear Algebra and its Applications, 30(0):69 - 75, 1980.

[11] J. K. Hale. Theory of functional differential equations, volume 3 of Applied Mathematical Sciences. Springer Verlag: New York, 1977. 
[12] J. K. Hale and S. M. Verduyn Lunel. Introduction to functional differential equations, volume 99 of Applied Mathematical Sciences. Springer Verlag: New York, 1993.

[13] R. Hryniv and P. Lancaster. On the perturbation of analytic matrix functions. Integral Equations and Operator Theory, 34:325-338, 1999.

[14] E. Jarlebring, K. Meerbergen, and W. Michiels. A Krylov method for the delay eigenvalue problem. SIAM Journal on Scientific Computing, 32(6):3278-3300, 2010.

[15] P. Kunkel and V. Mehrmann. Differential-Algebraic Equations: analysis and numerical solution. Textbook in Mathematics. EMS Publishing House, 2006.

[16] P. Lancaster, A.S. Markus, and F. Zhou. Perturbation theory for matrix function: the semisimple case. SIAM Journal of Matrix Analysis and Applications, 25(3):606-626, 2003.

[17] H. Langer and B. Najman. Remarks on the perturbation of analytic matrix functions II. Integral Equations and Operator Theory, 12:392-407, 1989.

[18] H. Langer and B. Najman. Remarks on the perturbation of analytic matrix functions III. Integral Equations and Operator Theory, 15:796-806, 1992.

[19] H. Langer and B. Najman. Leading coefficients of the eigenvalues of perturbed analytic matrix functions. Integral Equations and Operator Theory, 16:600-604, 1993.

[20] G. G. Lorentz and K. L. Zeller. Birkhoff interpolation. SIAM Journal on Numerical Analysis, 8(1):pp. 43-48, 1971.

[21] W. Michiels, K. Engelborghs, P. Vansevenant, and D. Roose. The continuous pole placement method for delay equations. Automatica, 38(5):747-761, 2002.

[22] W. Michiels and S.-I. Niculescu. Stability, Control, and Computation for Time-Delay Systems: An Eigenvalue Based Approach. Advances in Design and Control. SIAM Publications, Philadelphia, 2014.

[23] K. Schreiber. Nonlinear eigenvalue problems: Newton-type methods and nonlinear Rayleigh functionals. PhD thesis, TU Berlin, 2008.

[24] A.P. Seyranian and A.A. Maylibaev. Multiparameter stability theory with mechanical applications, volume 13 of Series on Stability, Vibration and Control of Systems. World Scientific, Singapore, 2003.

[25] F. De Téran. On the perturbation of singular analytic matrix functions: A generalization of Langer and Najman's results. Operators and Matrices, 5(4):553-564, 2011.

[26] F. Tisseur, K. Meerbergen, and Francoise Tisseur. A survey of the quadratic eigenvalue problem. SIAM Review, 43:2001, 2000.

[27] J. Vanbiervliet, K. Verheyden, W. Michiels, and S. Vandewalle. A nonsmooth optimization approach for the stabilization of linear time-delay systems. ESAIM: Control, Optimisation and Calcalus of Variations, 14(3):478-493, 2008.

[28] H. Voss. Nonlinear eigenvalue problems. In L. Hogben, editor, Handbook of Linear Algebra, chapter 60. CRC Press, 2014.

[29] J. Wilkening. An algorithm for computing Jordan chains and inverting analytic matrix functions. Linear Algebra and Its Applications, 427(1):6-25, 2007.

[30] Z. Wu and W. Michiels. Reliably computing all characteristic roots of delay differential equations in a given right half plane. Journal of Computational and Applied Mathematics, 236:2499-2514, 2012.

Appendix A. Functional Birkhoff/confluent Vandermonde matrices. Initially, Birkhoff and Vandermonde matrices were derived from the problem of polynomial interpolation of some unknown function $g$. This can be presented in a general way by describing the interpolation conditions in terms of incidence matrices, see for instance [20]. For given integers $n \geq 1$ and $r \geq 0$, matrix

$$
\mathcal{E}=\left(\begin{array}{ccc}
e_{1,0} & \ldots & e_{1, r} \\
\vdots & & \vdots \\
e_{n, 0} & \ldots & e_{n, r}
\end{array}\right)
$$

is called an incidence matrix if $e_{i, j} \in\{0,1\}$ for every $i$ and $j$. Such a matrix contains the data providing the known information about the function $g$. Let $x=$ $\left(x_{1}, \ldots, x_{n}\right) \in \mathbb{R}^{n}$ such that $x_{1}<\ldots<x_{n}$, the problem of determining a polynomial $\hat{P} \in \mathbb{R}[x]$ with degree less or equal to $\iota\left(\iota+1=\sum_{1 \leq i \leq n, 1 \leq j \leq r} e_{i, j}\right)$ that interpolates $g$ at $(x, \mathcal{E})$, i.e., which satisfies the conditions

$$
\hat{P}^{(j)}\left(x_{i}\right)=g^{(j)}\left(x_{i}\right),
$$


is known as the Birkhoff interpolation problem. Recall that $e_{i, j}=1$ when $g^{(j)}\left(x_{i}\right)$ is known, otherwise $e_{i, j}=0$. Furthermore, an incidence matrix $\mathcal{E}$ is said to be poised if such a polynomial $\hat{P}$ is uniquely defined.

A functional confluent Vandermonde matrix $\Phi$ is a matrix with the following structure:

$$
\left\{\begin{aligned}
\Phi & =\left[\Phi_{1} \Phi_{2} \ldots \Phi_{M}\right], \\
\Phi_{i} & =\left[f\left(\sigma_{i}\right) f^{(1)}\left(\sigma_{i}\right) \ldots f^{\left(d_{i}-1\right)}\left(\sigma_{i}\right)\right], \\
f\left(\sigma_{i}\right) & =g\left(\sigma_{i}\right) \cdot\left[1 \ldots \sigma_{i}^{l-1}\right]^{T}, \quad \text { for } \quad 1 \leq i \leq M
\end{aligned}\right.
$$

for a sufficiently regular function $g \in \mathcal{C}^{k}(\mathbb{R})$, see [10]. If, $g(x)=1$ then we are dealing with the so-called confluent Vandermonde matrix, see [10]. If, additionally, $d_{i}=1$ for $i=1 \ldots N$, then we recover the classical Vandermonde matrix. 